\title{
Ready for take-off
}

\author{
Antoine Müller and Jérôme Faist
}

\section{Since their invention, quantum cascade lasers have made considerable progress in terms of their wavelength range and efficiency. Today, they have important applications in environmental science, process control and medical diagnostics.}

The first demonstration of the quantum cascade laser (QCL) in 1994 came somewhat as a surprise. A semiconductor laser based on inter-subband transitions (energy levels within a single band) had been proposed by Kazarinov and Suris as early as 1972 . However, it was widely believed that the extremely short upperstate lifetime of electrons in quantum wells, although not suppressing laser operation completely, would impede the realization of a QCL laser with a performance suitable for practical applications.

Luckily, this turned out not to be the case, and the inter-subband transition approach of operation was shown to have some very attractive features. First, the wavelength could be adjusted over a very wide range from the mid- to farinfrared at the design stage by simply scaling the quantum well thicknesses. Second, the transitions could be cascaded over a large number of well periods in such a way that a single electron passing through the structure can emit a large number of photons in the process (10-100, in practice). In addition, growth and processing technologies already developed for telecommunications lasers were capable of fabricating this new device.

In any kind of laser, the threshold for lasing occurs when the gain balances the optical losses. The very short upper-state lifetime (subpicosecond) in the first QCLs meant that the gain would be very limited. However, this drawback was offset to a large extent by low waveguide losses and a low sensitivity of the gain to temperature. As a result, two key milestones demonstrating the potential of QCLs for applications were achieved a few years after its first operation. In 1997, pulsed singlefrequency operation at room temperature was achieved, and in 2002 continuous-wave operation was demonstrated.

Since then, considerable progress has been made. Today, QCLs have been fabricated to operate over a very wide wavelength range of 2.9-250 $\mu \mathrm{m}$, spanning the mid-infrared and terahertz regions. Furthermore, these devices operate at room

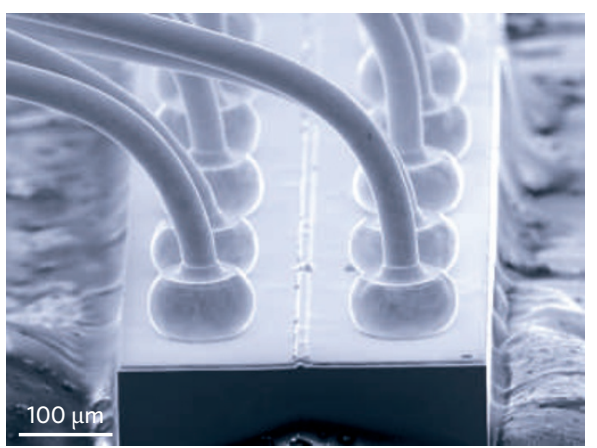

Scanning electron micrograph of a high-power QCL soldered on its submount. The waveguide ridge is visible between the rows of bonding wires, which bring current to the device.

temperature throughout a large portion of this frequency range. Devices operating in continuous-wave mode with $3 \mathrm{~W}$ of optical power and wall-plug efficiencies above $10 \%$ have been achieved, thanks to the low waveguide losses. Owing to the low temperature-sensitivity of the gain coefficient, single-mode devices operating in continuous-wave mode at temperatures of up to $150{ }^{\circ} \mathrm{C}$ have also been realized. Unlike conventional semiconductor lasers based on inter-band transitions, active regions operating at different wavelengths can be combined in a single waveguide, enabling the creation of QCLs with very broadband operation. Recently, a room-temperature device with a tuning of over $435 \mathrm{~cm}^{-1}$ was achieved, which corresponds to a bandwidth of $40 \%$ of the centre frequency. A number of companies are now selling devices with performances approaching these results.

As for applications, a large number of molecules have their fundamental vibrational mode in the mid-infrared region. This makes optical sensing and spectroscopy with QCLs a very powerful technique that combines both selectivity and absolute sensitivity. In contrast with other sensing techniques based on reactive membranes or surfaces, optical sensing is relatively immune to problems arising from chemical cross-talk or poisoning. Because QCLs are the first semiconductor laser sources to operate over the whole mid-infrared range at room temperature, they have enabled the development of sensing techniques that were previously limited by a lack of convenient optical sources. Applications of these optical sensing techniques are in areas such as environmental monitoring, process and combustion control, oil and gas industries, and medical diagnostics. As an example, a QCL-based spectrometer is due to be launched into space in the next few years to probe the isotopic content of the atmosphere on Mars.

Back on Earth, QCL-based spectrometers are also being used to provide continuous sampling of the $\mathrm{C}^{12} / \mathrm{C}^{13}$ and $\mathrm{O}^{16} / \mathrm{O}^{18}$ isotope ratios in the atmosphere. These instruments surpass mass-spectrometer-based instruments in terms of performance, and help to trace the sources and sinks of atmospheric $\mathrm{CO}_{2}$. Monitoring the exhaust gases of ships is an example of how combustion diagnostics is now being implemented.

In addition, QCL-based sensors promise to bring about a shift in the way sensing is performed, as they allow a simple change in optical source to address a different chemical compound.

Quantum cascade lasers are used in many other application areas, and the spread of the technology will be further increased by future advances in performance, such as ultralow dissipation to allow long-life battery-operated systems; increased wavelength ranges and broadly tunable devices; and terahertz operation with thermoelectric coolers. Increasing production volumes will also reduce the price of the technology, making it highly competitive with existing techniques.

Antoine Müller is CEO of Alpes Lasers, 1 Pass. Maxde-Meuron, Neuchâtel, Switzerland; Jérôme Faist is a professor at ETH Zurich, 8092 Zurich, Switzerland. e-mail:antoine.muller@alpeslasers.ch; jerome.faist@phys.ethz.ch 